\title{
ESTUDIO DE LA COMPOSICIÓN QUÍMICA DE LA MADERA DE CIPRÉS (Cupressus lusitanica) EN RELACIÓN AL FUSTE ${ }^{(*)}$
}

\author{
SANDRA RODRIGUEZ S. ${ }^{(* *)}$ y MARCO TORRES U. ${ }^{(* *)}$
}

(๑) Proyecto F-90 financiado por la Dirección de Investigación y Desarrollo, Universidad Austral de Chile

${ }^{(*)}$ Ingeniero Civil Químico

(*) Técnico en celulosa y papel.

Instituto de Tecnologia de la Madera, Facultad de Cienciaş Forestales, Universidad Austral de Chile, Casilla 567, Valdivia.

\section{RESUMEN}

El objetivo de este trabajo fue estudiar la variación de la composición química de la madera de Cupressus lusitanica en función de su posición en la dirección radial y apical en el fuste.

Se recolectaron 10 árboles de 2 parcelas, de las que se obtuvieron rodelas en las zonas basal, media y apical. De ellas se extrajeron muestras cada 6 anillos, desde la médula hacia la corteza.

El contenido de extraibles dismimuve desde médula a corteza y de la base al ápice en el árbol. El porcentaje de holocelulosa aumenta de médula a corteza y con la altura en el árbol obteniéndose un valor medio de $66 \%$. El contenido de lignina disminuye desde la médula a la corteza y desde la base al ápice, su valor medio fue de $33 \%$.

La composición quimica de la madera de ciprés permite esperar que no presente inconvenientes para ser pulpada a través de un proceso quimico normal. y que está dentro de los mángenes habituales para coniferas, destacándose su bajo contenido de extraibles y el alto contenido de lignina.

Palabras Clave: Composicion Quimica de la IIadera, Cupressus lusitanica 


\section{ABSTRACT}

The study aimed at the determination of the chemical composition of the wood of Cupressus lusitanica variation related to its position on the stem.

10 stems from two stands were collected, and wood specimens were taken at about base. middle and top heights of the trees and each six rings from the pith to the bark.

The extractive content decreases from the pith to the bark and at increasing height on the tree. The holocellulose content increases from the pith to the bark and with the height on the tree, showing an average value of $66 \%$. The lignin content decrease from the pith to the bark and from the base to the top; the average value obtained was $33 \%$.

The chemical composition of the wood indicates that it should not present problems to be pulped through a normal chemical process, and it is in the range found in conifers, remarking the low extractive and high lignin content of the species.

Keywords: Hood Chenical Composition, Cupressus Iusitanica 


\section{INTRODUCCIÓN}

En Chile se han introducido diferentes especies forestales de rápido crecimiento. especialmente coniferas, entre las que se destacan pino insigne (Pinus radiata D. Don) y pino oregón (Pseudotsuga menziessi (Mirb.), Franco). Junto a ellas existen otras que se han considerado como posibles alternativas, aún cuando no han alcanzado tasas de forestación importantes. Tal es el caso de ciprés mexicano (Cupressus lusitanica Mill.), cuya área de distribución natural incluye México y Guatemala (FAO, 1980; Vásquez, 1984).

La utilización del recurso existente y el incremento de las superficies reforestadas con esta especie, estrían sin duda relacionados con las características tecnológicas de la madera que se obtiene de ella en Chile. De ahí el interés por estudiar la propiedades y características de la madera de ciprés que crece en el país, y sus aptitudes como material de pulpaje.

Desde hace algún tiempo, se sabe de la viabilidad de usar la madera de C. lusitanica para producir celulosa. En Brasil existe un uso determinado de la especie para la producción de celulosa al sulfito de calidad comercial. A pesar de ello son pocos los antecedentes que se encuentran en la literatura.

Foelkel y Zvinakevicius (1978) dentro de su estudio de madera de especies exóticas para la producción de celulosa kraft incluyeron la de $\mathbf{C}$. lusitanica. Los autores determinaron una densidad básica en el DAP de $0,381 \mathrm{~g} / \mathrm{cm}^{3}$ y una composición química con un bajo contenido de extraíbles siendo los solubles en agua caliente y alcohol-benceno del orden de $1,36 \%$, solubles en soda al $1 \%$ de $9,5 \%$ y los porcentajes de holocelulosa y lignina de $72 \%$ y $34 \%$, respectivamente.

Palmer et al. (1986) determinaron las características pulpables de dos muestras de C. Iusitanica de 10 y 29 años de edad crecidas en una región de la India. Estas registraron una densidad de unos $400 \mathrm{~kg} / \mathrm{m}^{3}$ y fueron pulpadas mediante el proceso sulfato sin dificultad alguna. La madera presentó bajos contenidos de extraíbles en agua y etanol-benceno, siendo éstos del orden del 3\%. Los contenidos holocelulosa y de ligninafueron de $62 \%$ y $32 \%$. respectivamente.

En la sección transversal de un fuste. la madera presenta diferentes propiedades y características a través de su ubicación en el radio. La madera más cercana a la médula, llamada madera juvenil, presenta en coniferas menor densidad y largo de traqueidas, mayor ancho de anillos y ángulo fibrilar, y otras diferencias si se compara con la madera madura más alejada del centro del árbol. Estas provienen principalmente del efecto que cjerce la cercania de la copa en el cambium. La influencia se pierde a medida que crece el árbol y la copa se aleja de una determinada zona cambial, la que deja de formar madera juvenil. Las diferencias de comportamiento tecnológico entre la 
madera juvenil y madura tienen especial importancia en coniferas de crecimiento rápido. El volumen de madera juvenil en estas especies es muy importante, ya que su producción es mayor a medida que disminuye la edad de rotación y en casos llega a un $100 \%$ si se emplean las dimensiones menores de los árboles o el producto de raleos (Diaz-Vaz y Ojeda, 1980).

El objetivo del trabajo consistió en estudiar la variación en composición química de la madera de ciprés (C. lusitanica), en relación a su posición en el fuste del árbol.

\section{MATERIAL Y MÉTODO}

El material empleado es parte del utilizado en el estudio tecnológico de la especie, llevado a cabo en el Instituto de Tecnología de la Madera (Vásquez, 1984).

Para el desarrollo del estudio se dispuso de las plantaciones de C. lusitanica del Fundo Las Palmas ubicado a $15 \mathrm{~km}$ de Valdivia, X Región, Chile, de donde se seleccionaron dos parcelas ( 1 y 2 ) de edades entre 26 y 28 años.

Debido a que se iniciaron diversos estudios en forma conjunta, la totalidad de los árboles seleccionados una vez volteados fueron divididos de acuerdo a las necesidades de cada caso en particular. De este modo, para el presente trabajo en cada parcela se seleccionaron 5 árboles, de los cuales se obtuvieron rodelas en las zonas basal, media y apical del fuste, designadas como alturas 1,3 y 5 , respectivamente, las cuales permanecieron almacenadas en el Laboratorio de Pulpa y Papel del Instituto hasta la realización de este trabajo.

A partir de las rodelas se obtuvieron muestras cada 6 anillos en cada una de las tres alturas. En la dirección de médula a corteza éstas correspondieron a: 1 (0-6 anillos), 2 (7-12 anillos), 3 (13-18 anillos) y 4 (19-24 anillos), de donde se obtuvo un total de 11 muestras por árbol (considerando sentido radial y apical).

Desde las muestras obtenidas se obtuvieron astillas en forma manual con dimensiones aproximadas de 1 pulgada de largo, 3/4 pulgada de ancho y $4 \mathrm{~mm}$ de espesor. En seguida las astillas se almacenaron para su posterior procesamiento.

A cada muestra se le determinó su composición química de acuerdo a los siguientes procedimientos:

- Solubles en:

Etanol-Tolueno TAPPI T 204 om-88

$\mathrm{NaOH} 1 \% \quad$ TAPPI T $212 \mathrm{om}-88$

Agua caliente TAPPI T 207 om-88

Agua fria

- Cenizas

TAPPI T 207 om-88

- Lignina

TAPPI T 211 om- 88

- Holocelulosa

TAPPI T 222 om- 88

Poljak (Navarro. 1970) 


\section{RESULTADOS Y DISCUSIÓN}

El Cuadro $\mathrm{N}^{\circ} 1$ presenta la composición química promedio de los cinco árboles provenientes de cada parcela. Los valores promedio concuerdan con los obtenidos por otros autores para la especic (Foelkel y Zvinakevicius (1978). Palmer et al. (1986)). Se destacan su bajo contenido de extraibles y su alto contenido de lignina.

En las Figuras 1 a la 8 se muestra la variación de la composición química en relación a la posición en el fuste.

Los solubles en etanol-tolueno disminuyen en dirección radial desde médula a corteza y en dirección apical desde la base al ápice. En la parcela 2, en las alturas basal y media y a partir del $3^{\text {er }}$ grupo de anillos desde médula a corteza, se observa un aumento en cl contenido de extraibles.

Los valores en agua caliente y agua fría (Figura $\mathrm{N}^{\circ} 2$ y 3 ), disminuyen en dirección radial desde médula a corteza y en dirección apical desde la base al ápice. En los árboles de la parcela 2 este comportamiento se mantiene sólo hasta el $3^{\text {er }}$ grupo de anillos. a partir del cual el contenido de solubles aumenta en ambas direcciones.

\section{Cuadro $\mathrm{N}^{\circ} 1$}

\section{COMPOSICION QIINICA PROMEDIO DE Cupressus lusitanica}

\begin{tabular}{|l|r|r|}
\hline $\begin{array}{c}\text { Composición } \\
\left({ }^{\circ} \text { bms }\right)\end{array}$ & PARCELA 1 & PARCELA 2 \\
\hline Solubles en: & & \\
Etanol-Tolueno & 2.20 & 1.70 \\
Desv. Fstandar & 0,90 & 0.80 \\
Soda $1^{\circ}$ o & 12.80 & 13.00 \\
Desv. Fstandar & 1.60 & 1.30 \\
Agua Caliente & 2.30 & 2.30 \\
Desv. Estandar & 0.40 & 0.50 \\
Agua fria & 1.50 & 1.40 \\
Desv. Fstandar & 0.20 & 0.20 \\
Cenizas & 0.28 & 0.30 \\
Desv. Estandar & 0.07 & 0.06 \\
Lignina & 32.40 & 33.10 \\
Desv. Estaindar & 1.20 & 1.00 \\
Holocelulosa & 67.90 & 64.90 \\
Desv. Estándar & 1.50 & 1.20 \\
\hline
\end{tabular}




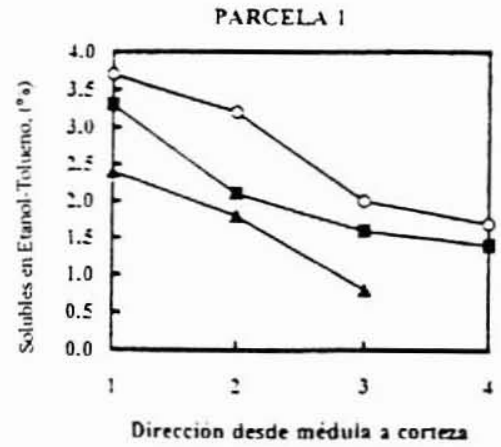

|a)

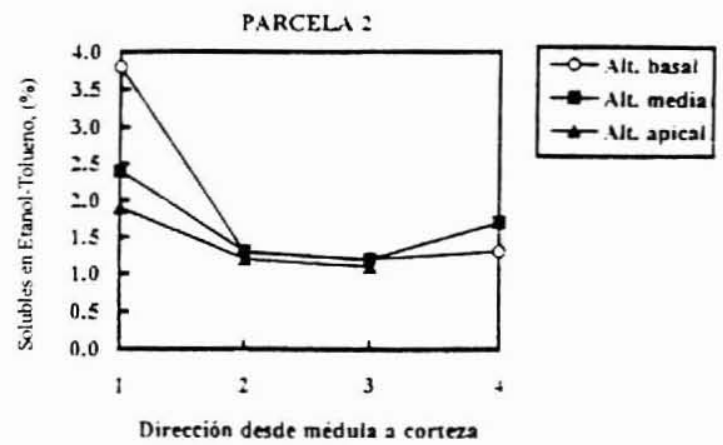

(b)

Figura $\mathrm{N}^{\circ} 1$. VARIACIÓN DEL CONTENIDO DE SOLUBLES EN ETANOL-TOLLIENO CON LA POSICIÓN EN EL FUSTE.

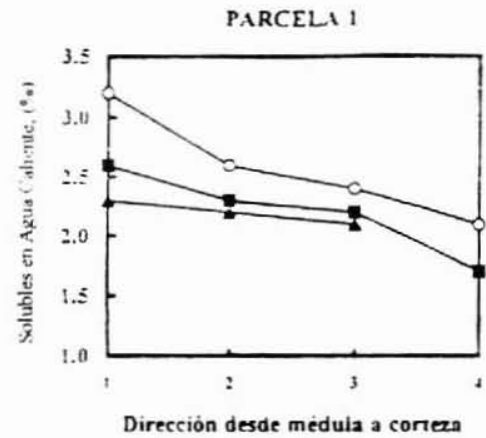

(a)

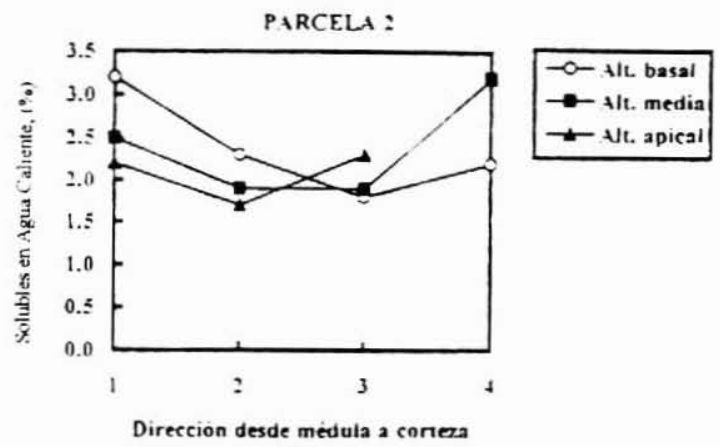

(b)

Figura $N^{\circ} 2$. VARIACIÓN DEL CONTENIDO DE SOLUBLES EN AGIAA CALIENTE CON LA POSICIÓN EN ELI. FISTE. 


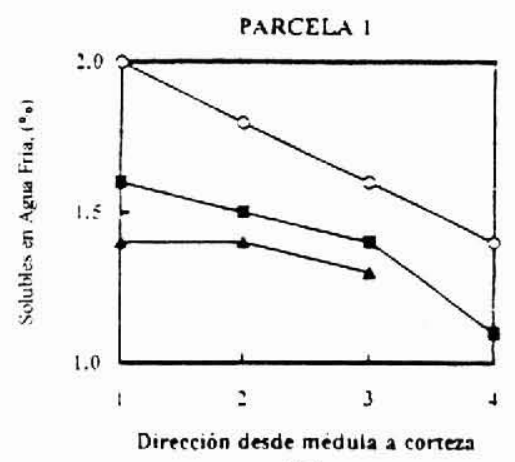

(a)
PARCELA 2

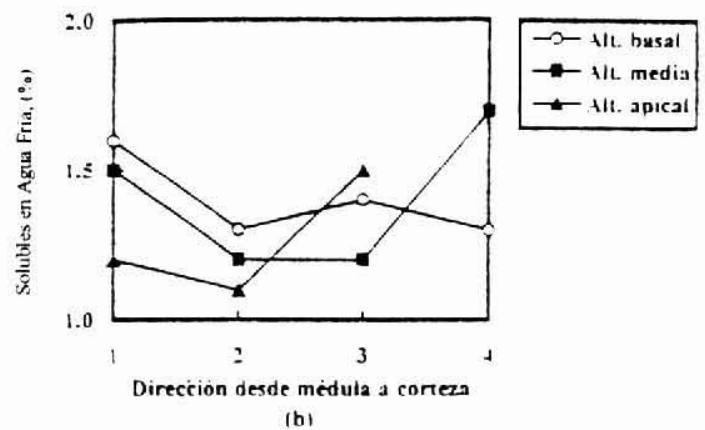

Figura $N^{\circ} 3$, VARIACIÓN DEL CONTENIDO DE SOLUBLES EN AGUA FRIA CON LA POSICIÓN EN EL, FUSTE.

Los solubles en soda $1 \%$ disminuyen en dirección radial de médula a corteza y en dirección apical desde la base al ápice para los árboles de la parcela 1. en tanto que en la parcela 2 nuevamente el comportamiento se invierte a partir del $3^{\text {er }}$ grupo de anillos (Figura $\mathrm{N}^{\circ} 4 \mathrm{~b}$ ).

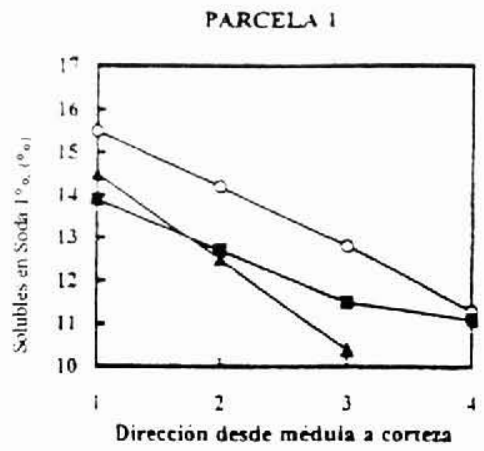

(a)
PARCELA 2

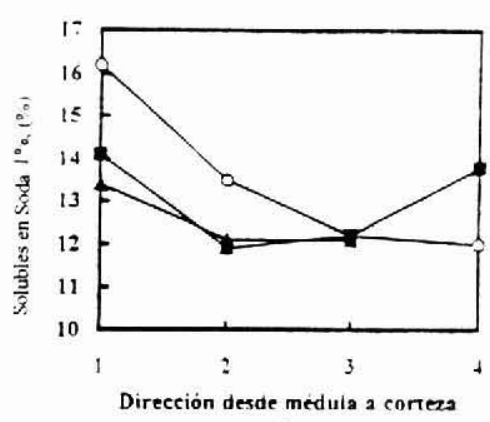

(b)

Figura $N^{\circ} 4$. VARIACIÓN DEL CONTENIDO DE SOLUBIES EN SODA $1 \%$ CON LA POSICIÓN EN EL FUSTE. 
El contenido de holocelulosa (Figura $\mathrm{N}^{\circ}$ ) aumenta en dirección radial de médula a corteza y en dirección apical desde la base al ápice.

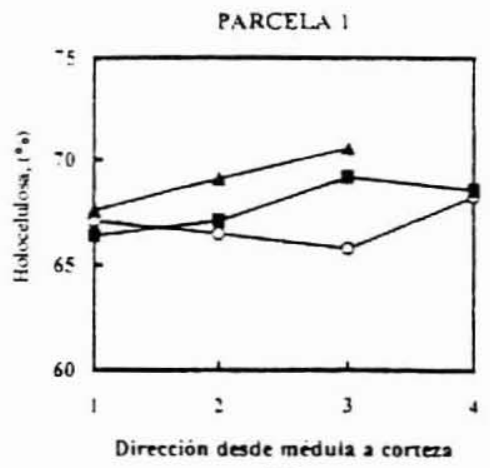

(a)

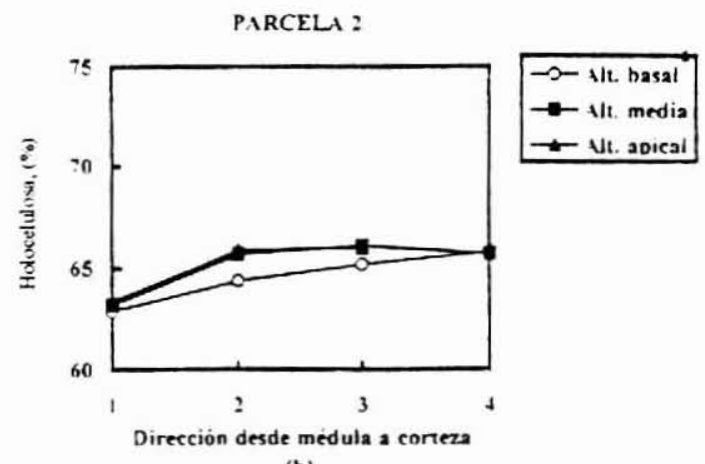

(b)

Figura N5. VARIACIÓN DEI, CONTENIDO DE HOLOCELLLOSA CON LA POSICIÓN EN EL FISTE.

En la Figura $\mathrm{N}^{\circ} 6$ se muestra el contenido de lignina. que disminuye en la dirección radial de médula a corteza y en dirección apical desde el ápice a la base. En la parcela 2 el contenido de lignina tiende a aumentar entre $\mathrm{cl} 2^{\mathrm{do}}$ y $3^{\text {er }}$ grupo de anillos (Figura $\mathrm{N}^{\circ} 6 \mathrm{~b}$ ).

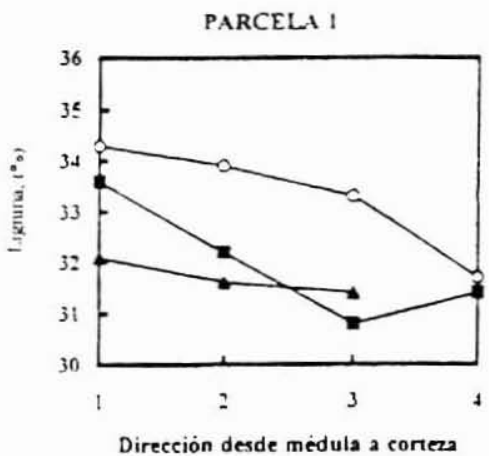

(a)

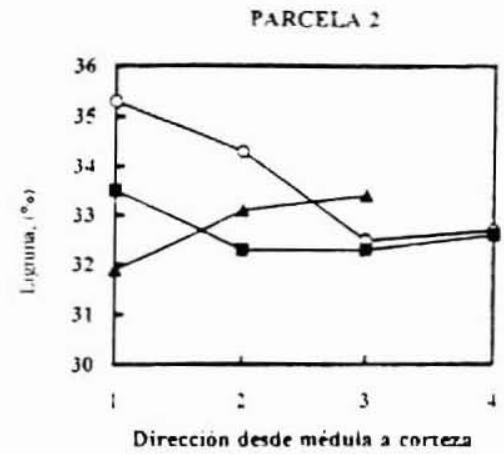

(b)

Figura $\mathrm{N}^{\circ} 6$. VARIACIÓN DE. CONTENIDO DF. I.IGNINA CON LA POSICIÓN EN EL FISTE. 


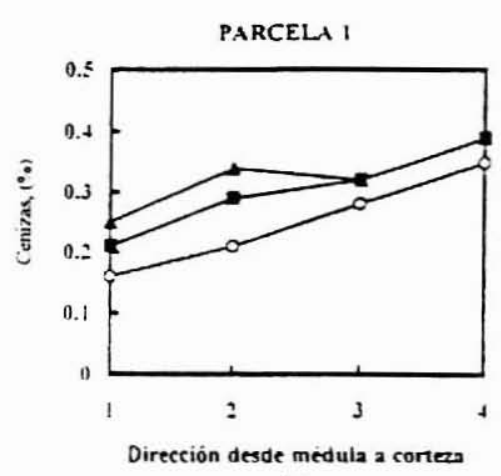

(a)

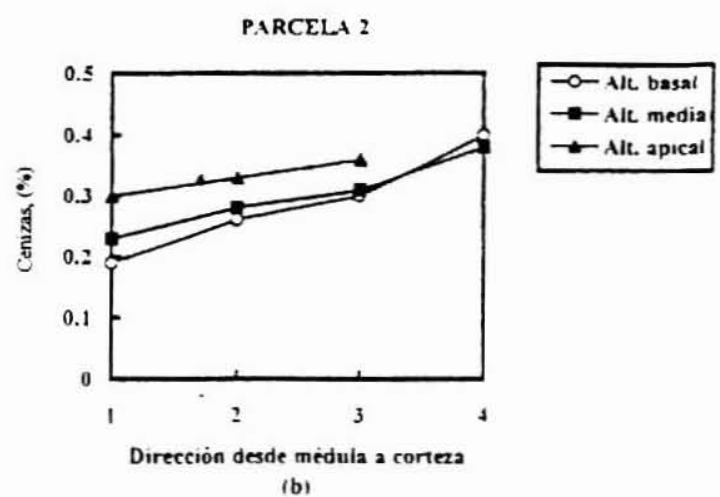

(b)

Figura Nº7. VARIACIÓN DEL CONTFNIDO DE CENIZAS CON LA POSICIÓN EN EL FUSTE.

El contenido de cenizas (Figura $\mathrm{N}^{\circ} 7$ ) aumenta de médula a corteza y con la altura en el árbol. para las dos parcelas. Esto es debido a que los compuestos inorgánicos participan en la función metabólica del árbol. la cual se desarrolla principalmente en la zona de la albura.

\section{CONCLUSIONES}

En general la madera de ciprés muestra una variación en composición química similar a la observada en otras coníferas. en relación a la posición en el fuste (Uprichard. 1971).

Con respecto a la composición química. entre las distintas parcelas no se observaron diferencias significativas. pero si hubo un comportamiento diferente en relación a la posición en el fuste. En la parcela 2 se produjo una diferencia en todas las propiedades del $3^{\text {er }}$ grupo de anillos desde médula a corteza. y dadas las características de esta porción de madera, cabe suponer que en la parcela 2 hubo presencia de madera de reacción o compresión. aun cuando no se realizaron ensayos físicos. En el caso de las coníferas la madera de compresión tiene mayor contenido de extraibles y lignina y menor contenido de celulosa que la madera normal.

Los valores de composición quimica son normales y se encuentran dentro de los margenes característicos para la clase. Destaca si el bajo contenido de extraíbles y el alto porcentaje de lignina presentes en la madera. como también fue determinado por otros autores (Foclkel y Zvinakevicius. 1978: Palmer et al., 1986). 
De esta manera es posible concluir que el $\mathbf{C}$. lusitanica no presenta complicaciones desde el punto de vista quimico para ser utilizado en la elaboración de pulpa quimica.

\section{REFERENCIAS BIBLIOGRAFICAS}

Diaz-Vaz, J.; Ojeda, F., 1980. Densidad Intraincremental de Pseudotsuga menziessi I Variaciones en un Análisis Fustal. Bosque, 3(2):86-95

FAO., 1980. Pulping and Paper-making of Fast Growing Plantation Wood Species, 1:101-103, Rome

Foelkel, C.; Zvinakevicius, C., 1978. Coniferas Exóticas Aptas para Producào de Celulose Kraft II "Cupressus lusitanica". CENIBRA pesquisa, (59). Belo Oriente. Cenibra

Navarro, J., 1970. Temas de la Fabricación del Papel. Editorial Marfil S A., Fspaña-Alcov, p 63

Palmer, E.; Gibbs, S.; Ganguli, S. and Dutta, A. P., 1986. Pulping Characteristics of Cupressus lusitanica and Podocarpus milanjanus Grown in the Sudan. Trop. Devt Res Inst. Rept. L73:23p.

Uprichard, J. M., 1971. Cellulose and Lignin Content in Pinus radiata D. Don. Within-Tree Variation in Chemical Composition, density and tracheid length. Holzforschung, 25(4):97-105.

Vásquez, F. R, 1984. Fibra revirada en Cupressus lusitanica (Mill) Crecido en Valdivia. Tesis de Grado, Facultad de Ciencias Forestales, Universidad Austral de Chile. 